\title{
On the Ways to Prevent Tennis Sports Injuries for Teenager Players
}

\author{
Xiying Zuo \\ Department of Sports, Henan University of Traditional Chinese Medicine, Zhengzhou City, 450000, \\ Henan Provence, China \\ Email: tuke126@126.com
}

Keywords: teenagers; tennis sports; prevention; sports injuries.

\begin{abstract}
As a fashionable sport and an entertainment, tennis is gaining more and more attention from people today. However, with its extensive development, the participants' injuries are not uncommon. This is especially true for teenager players who are easy to be injured in the tennis sports due to their ages. Accordingly, it is urgent to take some measures to reduce and prevent teenager injuries in the sports, which will not only help improve their sports achievements, but make positive impact on their future career and life.
\end{abstract}

\section{Introduction}

Tennis is a net resistance movement demanding higher physical fitness and strength, endurance, agility, speed, and other techniques of athletes. In addition, it represents sports of fashion combining competition fun and fitness in one nature, so it is loved by the majority of sports enthusiasts. In recent years, the number of sports enthusiasts that China has invested in it is growing, many schools also offer different levels of tennis lessons, and so how to reduce and prevent damage in teen tennis has become an important topic of discussion.

\section{Common injury of junior tennis player in the tennis}

Because tennis is different from other sports, its comprehensive physical fitness for athletes are higher, the athletes run around with a big margin, so the chances of causing movement damage would be relatively high. During exercise, the most common injury in adolescent athletes generally include muscle strain, ligament strain, sprain, tennis elbow, blisters, muscle cramps, shy sheath go far, and these injuries have not only a tremendous impact when they exercise, also cause great inconvenience to their life.

\section{Reasons of causing tennis teenagers injury}

\subsection{Technical movements error}

A good athlete must have beautiful and more accurate operation. The technical actions show not only the level of an athlete, but also play a vital role in their physical health. Correct actions promote the athlete level, errors or uncoordinated movements is the most prominent reason that led to sports injuries occur. Any one sport all experienced in the training process of athletes from all unfamiliar to the skilled process. As we all know, the movement is done by conditioned reflex, if in the process technology skills and to master action essentials is not enough, it is easy to produce wrong dynamic stereotype and causes a series of injuries to athletes' body. Junior tennis player in the growth and development of the growth period have strong ability to accept things and comprehend, after the formation of dynamic stereotype, it is difficult to change, so if it can not ensure the correctness of the action, and it can lead to sports injuries.

\subsection{Over local burden}

Over burden will lead to the accumulation of fatigue, causing movement strain. Tennis throughout the course of the campaign have a characteristic with a single action repetition that it needs to keep repeating the same action in the course of the campaign, if the practice time is too long, the number of 
repetitions you need also increases, this several times of repeated exercise will make muscles strong contract or over-stretched over the muscles that can bear the load, resulting in acute muscle strain or fracture; if repeated over-stretch of tendons and joints and lack of effective relaxation will become a strain. In addition, tennis needs to maintain body lean forward and knee in semiflexion state, this long process is likely to lead to knee knee strain, these are the causes that sports injuries occurr.

\subsection{Site equipment discomfort}

Site equipment discomfort is an objective reason leading athletes to cause sports injury. Because in tennis the stadium is small, time is faster, the number of participants are less, so it is not like basketball or soccer as an opportunity to rely on peers to complete in order to give yourself time to get rest. In addition, these features led to its certain limitations. Tennis players need better speed and power, in this case, if the site is too slippery, the athletes will be easy to fall, resulting in lower limb sprains, bruises, etc; if the site is too hard, the athletes during exercise, foot, ankle and knee, etc. get not effective buffer in the landing, causing more damage to the ankle knee.

\subsection{Insufficient preparation}

Preparation is one of the direct factors that lead to high and low probability of occurrence of sports injuries. For any one sport, preparatory activities are essential, to prepare activities are designed to enable athletes enter from the general state to motion, and it is the transition phase to make the players adapt to exercise intensity. Tennis belongs to net resistant and strenuous sport project, and in the course of the campaign, an athlete need to keep in endless running, moving, and other start state; if preparing activities are not full, in the course of the campaign cells is so viscous that muscle is in a stiff state and visceral functions can not sufficiently obtain mobilizes, so during exercise the occurrence of muscle strain, knee strain, sports injuries and cardiovascular aging is also not surprising.

\subsection{Other reasons}

Their physical factor is one of the factors causing the inevitable sports injuries, such as lack of muscle strength, poor ligament flexibility, incoordination, physical state of fatigue so easily leading to sports injuries. Juvenile psychological is not yet mature, in the course of the campaign often do not know how to adjust their status. When in poor health, they do not know make active and effective adjustment, thus resulting in injuries. In addition, the occurrence of sports injuries is also with athletes awareness about sports injuries, and many young people do not have a correct understanding of sports injuries and do not know how to protect themselves, these are the factors that increase the occurrence of sports injuries.

\section{How to prevent teen injury in tennis}

\subsection{Ensure the correctness of the action}

Correct technical movements can reduce the incidence of sports injuries. So playing ball is not only more coordinated, beautiful, but can also reduce the incidence of sports injuries. Therefore the junior tennis players at the beginner have the best guide of tennis coaches to learn the correct technical movements, to understand the law of tennis ball, in addition, beginners in the learning process should carefully ponder, put their experience into practice, and gradually improve the quality of their shots.

\subsection{Avoid local overburdening}

Movement load is decided by exercise time and exercise intensity, so in the prolonged practice process, we shall reasonably assign, as in practice we should shorten exercise intensity; we should shorten the exercise time when exercise intensity is too large. When in systematic practice, we should more arrange reasonably exercise force and exercise time to reduce the production of sports fatigue. Coaches can also arrange reasonably rest time according to the sport periodic character to effectively relax muscle and avoid exercise-induced fatigue.

\subsection{Fully prepared activities}

Preparation activities are primarily to reduce muscle viscosity and increase its mobility, flexibility and mechanical endurance, so that the athletes meet the needs of strenuous exercise. When carrying 
out preparatory activities is for the purpose of stretching muscles, ligaments, joints and adjusting the body internal organs to adapt the movement requirements, such as whole-body exercises, muscle stretching, jogging, joint activities as the main content.

\subsection{Choose the right sports equipment and inspect sites}

We should make reasonable choice for sports equipment, tennis supplies. During exercise, athletes should choose the right racket, tennis shoes and other items according to their own situation; choose protection support strip according to the easily hurt parts of sports such as ankle, wrist and other factors to ensure athletes from outside injury; inspect and maintain regularly or before training for sites so as not to cause harm during the exercise course.

\subsection{Improve full physical fitness}

Improve full physical fitness is one of the effective means to reduce sports injury. Young tennis athletes have not been yet fully developed and the force is relatively poor adult athletes, but there is another advantage of the speed and sensitiveness that adult athletes cannot surpass, so in the development of the tennis quality exercise, junior tennis players need to strengthen general physical training such as strengthening force, the joint strength and flexibility exercise that muscles sites are prone to injury to strengthen their power.

\subsection{Enhance students 'understanding of sports injuries, and improve students' self-protection awareness}

Enhancing understanding of sports injuries and improving students' awareness of self-protection can effectively prevent the occurrence of sports injuries. In Tennis Teaching, coaches should enhance students' understanding of sports injury, when in teaching students technical action essentials, also make certain guide for strengthening the importance of the students about the causes and prevention of sports injury, improving their initiative self-protection awareness .

\subsection{Strengthen the medical oversight}

In the tennis training process, coaches and students should strengthen the medical oversight and improve awareness of medical supervision to ensure that students can consciously applied to the sense of medical into practice. During tennis training, coaches should patiently explain precautions and arrangements to eliminate fatigue for students and relieve muscle relaxation exercises, so that they were in training make their own reasonable protection and training for their own body to make a series of adjustments for avoiding exercise fatigue accumulation.

\section{Conclusion}

Through the above analysis and discussion, we conclude that as a fashion physical fitness sports, tennis has been involved by more and more young people, and we should fully improve their awareness of injury prevention and precautionary measures. This is our responsibility and obligation. Especially tennis coaches should guide them in his teaching correctly understand the damage of tennis , strengthen norm teaching for the basic actions of beginners and the training of easily hurt parts to ensure that junior tennis players have a healthy system and can more actively and effectively involve in tennis.

\section{References}

[1] Huang Wangjun. Tennis enthusiasts prevent sports injuries [J]. Ningbo College journal, 2010 (6).

[2] Luan Lixia, Xu Xiangfeng. Research into college Students tennis injury [J]. Wuhan Institute of Physical Education, 2015 (6).

[3] Bao Qin. Common Tennis Sports Injury and Prevention [J]. The journal of Nanjing Institute of Physical Education, 2014 (6).

[4] Zhang Lu Ping. Sport Traumatology massage [M]. Guangxi Normal University Press, 2012. 
[5] Shao Yuping. Common injuries and preventive measures caused by tennis [J]. Hubei Sports Science, 2014 (4). 